\title{
Sequential release of salidroside and paeonol from a nanosphere-hydrogel system inhibits ultraviolet B-induced melanogenesis in guinea pig skin
}

\author{
This article was published in the following Dove Press journal: \\ International Journal of Nanomedicine \\ 16 April 2014 \\ Number of times this article has been viewed
}

\author{
Li-Hua Peng' \\ Shen-Yao Xu' \\ Ying-Hui Shan' \\ Wei Weil \\ Shuai Liu' \\ Chen-Zhen Zhang' \\ Jia-He Wu' \\ Wen-Quan Liang' \\ Jian-Qing Gao ${ }^{1,2}$ \\ 'Institute of Pharmaceutics, College \\ of Pharmaceutical Sciences, Zhejiang \\ University, Hangzhou, ${ }^{2}$ Novel \\ Transdermal Research Center of \\ Jiangsu Province, Changzhou, People's \\ Republic of China
}

\begin{abstract}
Melanin is the one of most important pigments for skin color in mammals. Excessive biosynthesis of melanin induces various pigment disorders. Much effort has been made to develop regulators to minimize skin pigmentation abnormalities. However, only a few of them are used, primarily because of safety concerns and low efficiency. In this study, we aimed to construct a novel nanosphere-gel for sequential delivery of salidroside and paeonol, to investigate the synergistic effects of these drugs in anti-melanogenesis, and to decrease their potential for toxicity in high dosage. Nanospheres were prepared and characterized for their particle size, polydispersity index, zeta potential, and morphological properties. The optimized nanospheres were incorporated in carbomer hydrogel with both paeonol and salidroside entrapped to form a dual drug-releasing nanosphere-gel. With this nanosphere-gel, rapid release of salidroside from the hydrogel followed by sustained release of paeonol from the nanosphere was achieved. Using a classical model of the melanogenesis response to ultraviolet exposure, it was shown that the anti-melanogenesis effects of the dual drug-releasing system, in which the doses of the individual drugs were decreased by half, was obviously enhanced when compared with the effects of the single drug preparations. Mechanistically, the burst release of salidroside from the hydrogel may enable prompt suppression of melanocyte proliferation on exposure to ultraviolet B radiation, while the paeonol released in a sustained manner can provide continuous inhibition of tyrosinase activity in melanocytes. Combined delivery of salidroside and paeonol was demonstrated to be a promising strategy for enhancing the therapeutic efficacy of these agents in anti-melanogenesis and reducing their toxicity, so may have great potential in nanomedicine.
\end{abstract}

Keywords: nanosphere, sequential drug release, anti-melanogenesis, salidroside, paeonol

\section{Introduction}

Melanin is the major pigment for color in human skin. It is secreted by melanocyte cells in the basal layer of the epidermis., ${ }^{1,2}$ Apart from contributing to cosmetic appearance, melanin functions as an effective biological barrier for absorbing free radicals from the cytoplasm ${ }^{3,4}$ and shielding from ultraviolet (UV) light., ${ }^{5,6}$ However, overproduction and accumulation of melanin can cause hyperpigmentation problems and skin disorders such as freckles, melasma, and lentigines. ${ }^{7-10}$ Aside from constitutive melanin synthesis, solar UV radiation is the main etiological factor in melanogenesis. ${ }^{11,12}$ Many factors that increase melanogenesis are upregulated in response to UV irradiation and result in hyperpigmentation. ${ }^{13}$ Failure to eliminate ultraviolet B (UVB)-damaged cells by controlled apoptosis may result
Correspondence: Jian-Qing Gao Institute of Pharmaceutics, College of Pharmaceutical Sciences, Zhejiang University, 866 Yuhangtang Road, Hangzhou 310058, People's

Republic of China

$\mathrm{Tel}+8657 \mid 88208437$

Fax +86 57। 88208437

Email gaojianqing@zju.edu.cn 
in pigmentation disorders induced by accumulation of melanin, such as melasma, freckles, ephelides, and senile lentigines. ${ }^{14,15}$

Briefly, UVB may play a critical role in the synthesis of melanin via two major mechanisms. First, it has a strong ability to stimulate proliferation of melanocytes and promote synthesis of melanin after sun exposure ${ }^{16}$ and, second, UVB stimulates tyrosinase activity in cells to produce melanin. ${ }^{17}$ Therefore, both inhibition of melanocyte proliferation and activation of tyrosinase are critical for anti-melanogenesis. In a previous study, we reported on two natural products, ie, paeonol and salidroside, which have anti-melanogenesis effects by inhibition of tyrosinase activity and melanocyte proliferation, respectively. ${ }^{18}$ Given that salidroside and paeonol can interfere with synthesis of melanin via several mechanisms, it is widely hypothesized that combined delivery of these compounds would be very useful for enhancing their therapeutic efficacy and would allow the drug doses to be decreased, thereby reducing their potential toxic effects. However, as far as we know, the evidence for a synergistic effect of multiple drugs in anti-melanogenesis is scarce. Therefore, the main aim of this study was to investigate the anti-melanogenesis efficacy of the combined delivery of paeonol and salidroside, in contrast with that of each drug administered alone.

Paeonol is a phenolic-type compound and almost insoluble in water, ${ }^{19,20}$ while salidroside is a glucoside of tyrosol and is highly soluble in water. ${ }^{21}$ Figure 1 shows the chemical structures of salidroside and paeonol. Combined delivery of these two compounds is not possible using simple drug solutions or conventional preparations. Further, salidroside decreases the proliferation of melanocytes, whereas paeonol downregulates tyrosinase activity in melanocytes, the delivery manner with a fast release of salidroside followed by the sustained release of paeonol can prevent the melanin synthesis from multiple pathways. The problems of short-term effects and poor stability, need for frequent administration, and poor penetration of the stratum corneum have greatly hampered application of drug solutions and conventional preparations in the clinic. ${ }^{22}$ The application frequency of salidroside and paeonol once daily may make these agents a good choice for the treat-

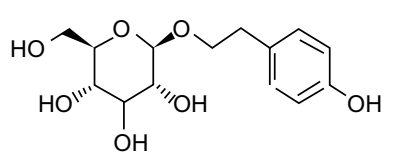

Salidroside

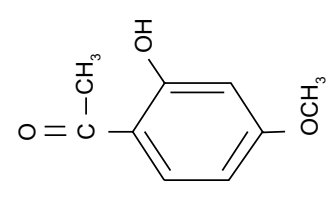

Paeonol
Figure I Chemical structure of salidroside and paeonol. ment of melanogenesis. A novel carrier is required to reduce the dosing frequency and to improve patient compliance. ${ }^{23}$ Accordingly, another aim of this study was to construct a novel nanotechnology-based system to deliver salidroside and paeonol sequentially, to protect the drugs from degradation, and to reduce their potential for toxicity at high dosage.

Topical drug delivery systems are particularly useful because systemic side effects can be reduced compared with parenteral or oral drug administration. ${ }^{24}$ Because of their ultrasmall and controllable size, large surface area to mass ratio, high reactivity, and functionalizable structure, ${ }^{25,26}$ various nanoscale technologies have been used to construct topical drug delivery systems allowing simultaneous delivery of multiple drugs. ${ }^{27-29}$ Specifically, lipid nanoparticle-based carriers that can attach to the skin surface and allow lipid exchange between the outermost layers of the stratum corneum and the carrier appear to be promising for improving penetration of the stratum corneum. ${ }^{30}$ Therefore, in this study, lipid nanospheres were optimized as carriers for sustained release of paeonol.

Another useful carrier, ie, water-based hydrogel, allows burst release of drug from the sheeting for a rapid effect, and the hydrogel can remain at the skin site with no need for other material for cover and/or fixation. ${ }^{31}$ In the present study, hydrogel was loaded with salidroside for rapid release of this compound. The hydrogel carrier was selected to provide an adhesive phase for the nanoparticle suspension, to increase the chemical stability of the drugs in the formulation, and to improve adhesion of the nanosuspension to the skin. Therefore, the optimized nanospheres were incorporated in a carbomer hydrogel entrapping salidroside to form a dual drug-releasing nanosphere-gel. Using this system, the intention was to provide burst release of salidroside from the hydrogel, which would rapidly inhibit synthesis of melanin in response to irritation by UVB, followed by continuous elimination of melanin by the paeonol released in a sustained manner from the nanospheres. The efficacy of this dual drugreleasing nanosphere-hydrogel in decreasing and preventing melanogenesis was investigated using the UVB-irradiated melanogenesis skin model, ${ }^{32,33}$ with single drug-loaded preparations serving as controls.

\section{Materials and methods Materials}

Paeonol and salidroside ( $>99.5 \%$ purity) were purchased from the Zhejiang Institute of Food and Drug Control (Zhejiang, People's Republic of China). Compritol ${ }^{\circledR}$ ATO 888 was provided by Gattefosse (Shanghai, People's Republic of China), 
Dynasan $^{\circledR} 116$ was purchased from Contensio Chemicals GmbH (Witten, Germany), and clotrimazole and Miglyol ${ }^{\mathbb{R}}$ 812 were obtained from Caelo (Hilden, Germany). Xanthan gum was sourced from Sigma-Aldrich (St Louis, MO, USA). Lecithin (Sigma-Aldrich) and Tween-80 (Shanghai Chemical Co, Ltd, Shanghai, People's Republic of China) were chosen as surfactants. Carbopol ${ }^{\circledR}$ 934, 940, and 941 was provided by Hangzhou Keben Chemical Co, Ltd (Zhejiang, People's Republic of China). Double-distilled water was used in all experiments. All other chemicals and solvents were of analytical reagent grade. A Masson's trichrome staining kit was purchased from Nanjing Keygen Inc. (Nanjing, People's Republic of China). Buffered paraformaldehyde (4\%) was purchased from Boster Inc. (Wuhan, People's Republic of China).

\section{Optimization of nanosphere formulation}

The nanospheres were prepared using the solvent evaporation method. Briefly, the lipid phase (consisting of solid-lipid and liquid-lipid materials) was melted with lecithin at $80^{\circ} \mathrm{C}$ to obtain a clear lipid phase. Meanwhile, an aqueous surfactant solution was prepared and heated to the same temperature. The hot surfactant solution was then dispersed in the hot lipid phase using a magnetic stirrer at 2,000 rpm for 30 minutes. The coarse hot oil-in-water emulsion obtained was ultrasonicated using a Sonopuls ultrahomogenizer (Bandelin, Berlin, Germany) for 20 minutes. A series of nanospheres was prepared by varying the types and contents of lipid, drug, and surfactant in water (Table 1), and evaluated for their size and zeta potential.

\section{Gels enriched with nanospheres}

The gels were prepared using four polymers, ie, Carbopol 934 (1\%), Carbopol 940 (1\%), Carbopol 941 (1\%), and xanthan gum $(1 \%)$. For preparation of the hydrogel, the gel-forming polymer was dispersed in double-distilled water overnight, and then neutralized with $\mathrm{NaOH}$. After adding the nanosphere dispersion (using the best formulation obtained by optimization), the sample was mixed using a high-speed stirrer at approximately $500 \mathrm{rpm}$ for 10 minutes. Finally, the $\mathrm{pH}$ of the sample was adjusted to $7.0 \pm 0.2$. The nanosphere-incorporated hydrogel was then stored at $4^{\circ} \mathrm{C}$ for up to 6 weeks.

\section{Characterization of the nanosphere dispersion and nanosphere-gel Particle size analysis and zeta potential}

To determine the size and zeta potential of the nanospheres, the nanosphere dispersion and nanosphere-gel were diluted with double-distilled water and then adjusted to a conductivity of $50 \mathrm{mS} / \mathrm{cm}$ with $\mathrm{NaCl}$. The measured electrophoretic mobility was converted to a zeta potential using the Helmholtz-Smoluchowski equation. This was done using the software included in the system.

\section{Morphological analysis by TEM}

The morphology of the nanospheres was observed by transmission electron microscopy (TEM), (JEM-1200EX, JEOL Ltd, Tokyo, Japan). The required sample was prepared by placing a drop of formulation diluted 50-fold with doubledistilled water onto a 400-mesh copper grid coated with

Table I Size, polydispersity index, and zeta potential of nanospheres

\begin{tabular}{|c|c|c|c|c|c|}
\hline \multirow[t]{2}{*}{ Number } & \multirow{2}{*}{$\frac{\text { Lipid phase }}{\text { Solid lipid/liquid lipid (w/w) }}$} & \multirow{2}{*}{$\frac{\text { Water phase }}{\text { Surfactant }}$} & \multicolumn{3}{|l|}{ Day 0} \\
\hline & & & Size $(\mathrm{nm})$ & PDI & ZP $(\mathrm{mV})$ \\
\hline I & ATO:MIG (7:3) & I\% T-80 & $452 \pm 23$ & $0.286 \pm 0.18$ & \\
\hline 2 & ATO:MIG (6:4) & $1 \% \mathrm{~T}-80$ & $3,940 \pm 17$ & $I \pm 0.2 \mathrm{I}$ & \\
\hline 3 & ATO:СCT (7:3) & $1 \% \mathrm{~T}-80$ & $584 \pm 44$ & $0.325 \pm 0.09$ & \\
\hline 4 & ATO:СCT (6:4) & $1 \% \mathrm{~T}-80$ & $800 \pm 38$ & $0.916 \pm 0.17$ & \\
\hline 5 & Dyna:CCT (7:3) & $1 \% \mathrm{~T}-80$ & $559 \pm 29$ & $0.542 \pm 0.21$ & \\
\hline 6 & Dyna:CCT (6:4) & $1 \% \mathrm{~T}-80$ & $4 \mid 2 \pm 55$ & $0.432 \pm 0.06$ & \\
\hline 7 & Dyna:MIG (7:3) & $1 \% \mathrm{~T}-80$ & $305 \pm 12$ & $0.300 \pm 0.13$ & \\
\hline 8 & Dyna:MIG (6:4) & I\% T-80 & $275 \pm 15$ & $0.208 \pm 0.25$ & $-15 \pm 4$ \\
\hline 9 & Dyna:MIG (5:5) & $1 \% \mathrm{~T}-80$ & $303 \pm 24$ & $0.205 \pm 0.05$ & \\
\hline 10 & Dyna:MIG (4:6) & I\% T-80 & $352 \pm 21$ & $0.227 \pm 0.36$ & \\
\hline II & Dyna:MIG (3:7) & $1 \% \mathrm{~T}-80$ & $328 \pm 32$ & $0.184 \pm 0.27$ & \\
\hline 12 & Dyna:MIG (6:4) & I\% lecithin & $322 \pm 19$ & $0.168 \pm 0.03$ & $-33 \pm 2$ \\
\hline \multirow[t]{2}{*}{13} & Dyna:MIG (6:4) & $0.5 \% \mathrm{~T}-80$ & $260 \pm 15$ & $0.232 \pm 0.04$ & $-26 \pm 5$ \\
\hline & & $+0.5 \%$ lecithin & & & \\
\hline
\end{tabular}

Notes: Data are expressed as the mean \pm standard deviation. ATO (Compritol ${ }^{\circledR}$ ATO 888; Gattefosse, Shanghai, People's Republic of China); Dyna (Dynasan ${ }^{\circledR}$ II 6 V; Contensio Chemicals GmbH, Witten, Germany); MIG (Miglyol ${ }^{\circledR}$ 8I2; Caelo, Hilden, Germany); CCT (Caprylic capric triglycerride; Hangzhou Jianghui Technology Co., Ltd, Hangzhou, People's Republic of China); T-80 (Tween-80, Shanghai Shize Biotechnology Co, People's Republic of China).

Abbreviations: PDI, polydispersity index; ZP, zeta potential. 
carbon film followed by negative staining with $1 \%$ phosphotungstic acid.

\section{Drug entrapment efficiency}

The amount of drug encapsulated per unit weight of the nanospheres was determined after separation of the free drug and lipids from the aqueous medium. A known dilution of the nanosphere dispersion was prepared, from which $100 \mu \mathrm{L}$ was transferred to the upper chamber of a Nanosep ${ }^{\circledR}$ centrifuge tube (Pall Life Sciences, Portsmouth, UK) fitted with an ultrafilter (molecular weight cutoff $100 \mathrm{kDa}$ ). The Nanosep tube was centrifuged at $12,000 \times \mathrm{g}$ using a TC 4100 D research centrifuge (Eltek, Mumbai, India) for 30 minutes. The filtrate was diluted appropriately and the amount of paeonol in both phases was estimated by high-pressure liquid chromatography. The percentage entrapment efficiency was calculated using the following equation:

$$
\left(\mathrm{W}_{\text {initial drug }}-\mathrm{W}_{\text {free drug }}\right) / \mathrm{W}_{\text {initial drug }} \times 100 \%
$$

where $\mathrm{W}_{\text {initial drug }}$ is the mass of the initial drug added and $\mathrm{W}_{\text {free drug }}$ is the mass of free drug detected in the filtrate of the lower chamber of the Nanosep tube following centrifugation of the aqueous dispersion.

\section{In vitro drug release}

A drug release study was performed using the dialysis method. A dialysis bag retains nanospheres and nanospheregel but allows transfer of dissolved/released drug molecules into the release medium. Briefly, dialysis bags (molecular weight cutoff 8,000-14,000 kDa) were prepared according to the protocol recommended by Sigma-Aldrich and then soaked in the release medium overnight prior to the study. The release medium was $1 \mathrm{mM}$ phosphate-buffered saline ( $\mathrm{pH}$ 7.4). The nanosphere dispersion and nanosphere-gel were put in the dialysis bags, which were placed in centrifuge tubes containing $25 \mathrm{~mL}$ of the release medium. The bottle was capped and placed on a shaking box at $37^{\circ} \mathrm{C}$ and $75 \mathrm{rpm}$. At predetermined time points, a $2 \mathrm{~mL}$ sample of the release medium was withdrawn from the bottle and replaced with $2 \mathrm{~mL}$ of fresh release medium to maintain the sink condition. The samples were analyzed by highpressure liquid chromatography. Paeonol was separated on an ODS C18 column $(250 \mathrm{~mm} \times 4.6 \mathrm{~mm}, 5 \mu \mathrm{m})$ and eluted with mobile phase of methanol-water $(60: 40, \mathrm{v} / \mathrm{v})$ at a flow rate of $1 \mathrm{~mL}$ per minute. Detection was done at $274 \mathrm{~nm}$. Salidroside was separated on a reversed phase C18 column
$(250 \mathrm{~mm} \times 4.6 \mathrm{~mm}, 5 \mu \mathrm{m})$ and eluted with mobile phase of methanol-water $(70: 30, \mathrm{v} / \mathrm{v})$ at a flow rate of $1 \mathrm{~mL}$ per minute. Detection occurred at $274 \mathrm{~nm}$.

\section{Stability of nanosphere-hydrogel}

Particle size and polydispersity index are important characteristics when evaluating the stability of colloidal systems, and were evaluated for the nanospheres during storage at $4^{\circ} \mathrm{C}$ for up to 28 days after their production.

\section{UVB-induced melanogenesis in brown guinea pigs}

Brown guinea pigs (weighing approximately 500 g) were supplied by Zhejiang University Experimental Animal Center (Zhejiang, People's Republic China). All animals were maintained under constant conditions (temperature $25^{\circ} \mathrm{C} \pm 1^{\circ} \mathrm{C}$ ) and had free access to a standard diet and drinking water. All of the experimental procedures were performed in accordance with the Zhejiang University guidelines for the welfare of experimental animals.

UVB-induced melanogenesis was induced on the backs of guinea pigs weighing approximately $500 \mathrm{~g}$. The animals were anesthetized with pentobarbital, and separate areas $(1.5 \mathrm{~cm} \times 1.5 \mathrm{~cm}$ square) on the back of each animal were exposed to UVB radiation (Waldmann UV 800, Herbert Waldmann GmbH \& Co, KG, Villingen-Schwenningen, Germany), with a TL/12 lamp emitting at 280-305 nm (Philips, Eindhoven, the Netherlands). The total UVB dose was $500 \mathrm{~mJ} / \mathrm{cm}^{2}$ per exposure. ${ }^{34}$ The animals were exposed to UVB radiation three times a week for 2 consecutive weeks. For the animal model experiments, the guinea pigs received only 2 consecutive weeks of UVB irradiation without any further handling.

For the pharmacological investigation, the animals were divided into four groups containing six animals each, as follows: a blank control group; a paeonol nanosphere-water suspersion group ( $1 \mathrm{~mL}$ of paeonol nanosphere dispersion, with $0.2 \mathrm{mg}$ of paeonol given every 2 days); a salidroside hydrogel group ( $1 \mathrm{~mL}$ of salidroside hydrogel, with $0.2 \mathrm{mg}$ of salidroside given every 2 days); and a paeonol and salidroside-coloaded nanosphere-hydrogel group (0.1 mg of paeonol and $0.1 \mathrm{mg}$ of salidroside given every 2 days). All drug preparations were administered topically for 2 consecutive weeks with simultaneous UVB exposure. Skin biopsies were taken and processed by histological analysis using Fontana-Masson staining. Figure 2 shows the basic design of the animal study. 


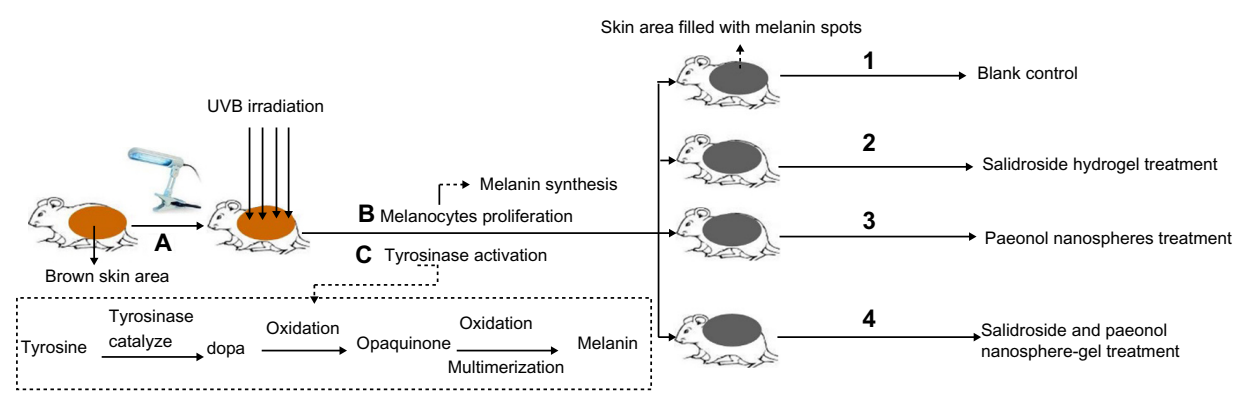

Figure 2 Flow chart of the animal study procedures. (A) UVB irradiation of the brown area in guinea pig skin. (B) Melanin synthesis caused by melanocyte proliferation upon UVB radiation. (C) Melanin synthesis caused by tyrosinase activation in melanocytes upon UVB radiation. (I) Blank control group: brown guinea pig skin without treatment. (2) Brown guinea pig skin treated topically with salidroside hydrogel every 2 days with simultaneous UVB radiation. (3) Irradiated brown guinea pig skin treated topically with paeonol nanosphere suspension every 2 days with simultaneous UVB radiation. (4) Irradiated brown guinea pig skin treated topically with salidroside and paeonol coloaded nanosphere-gel every 2 days with simultaneous UVB radiation.

Abbreviation: UVB, ultraviolet B.

\section{Histological analysis of guinea pig skin}

\section{Hematoxylin and eosin staining}

Skin biopsy specimens were fixed in $4 \%$ paraformaldehyde solution for 24 hours at $4{ }^{\circ} \mathrm{C}$. The tissue was then dehydrated and embedded in paraffin according to standard procedures. Serial $3 \mu \mathrm{m}$ thick sections were obtained. The histological properties of the skin were analyzed using hematoxylin and eosin staining. ${ }^{35}$

\section{Fontana-Masson staining}

Skin biopsy specimens were fixed in $4 \%$ paraformaldehyde solution for 24 hours at $4^{\circ} \mathrm{C}$. The tissue was then dehydrated and embedded in paraffin according to standard procedures. Serial $3 \mu \mathrm{m}$ thick sections were obtained. Melanocytes and melanin were visualized using FontanaMasson staining. ${ }^{36}$

\section{Statistical analysis}

The results are expressed as the mean $( \pm$ standard deviation) values of three experiments. The means were analyzed by Student's $t$-test using Statistical Package for the Social Sciences version 13 software (SPSS Inc., Chicago, IL, USA). $P$-values $<0.05$ were considered to be statistically significant.

\section{Results \\ Optimization of nanosphere formulation for paeonol}

A total of 14 experiments were performed (Table 1) that provided useful information about the main effects of the selected variables, including the types and percentages of the solid, liquid, and lipid materials, as well as the surfactants. The mean particle size and polydispersity index of colloidal carriers are important characteristics for nanospheres, from which the stability of drug-loaded nanospheres can be predicted. As shown in Table 1, using a combination of Compritol ATO 888 and Miglyol 812 or CCT (LABRAFAC CC, Shanghai Thanch Pharmaceuticals Technology Co, People's Republic of China) (samples 1-4), the prepared nanospheres were large in size with a high polydispersity index. In contrast, using Dynasan 116 as a solid lipid element, the particle size and polydispersity index of the nanospheres were markedly decreased (samples 5-13). The best formulation was obtained when the Dynasan 116 and Miglyol 812 were in a ratio of 6:4; ie, the smallest particle size was $275 \mathrm{~nm}$ with a low polydispersity index of 0.208 (sample 8 ). The surfactant used greatly affected particle size and polydispersity index.

It has been reported that combination of different types of surfactants is advantageous for decreasing particle size and increasing the stability of a nanosphere. ${ }^{37} \mathrm{In}$ the present study, $1 \%$ lecithin and a mixture containing $0.5 \%$ of T- 80 and $0.5 \%$ of lecithin were compared for their influence on the formation of nanospheres. It was found that the mixture containing $0.5 \%$ $\mathrm{T}-80$ and $0.5 \%$ lecithin had an advantageous effect in terms of decreasing the particle size and polydispersity index and increasing the stability (sample 13).

The zeta potential is also an important parameter predicting the stability of nanospheres as well as their potential mucoadhesive properties. Generally, a high surface charge reflects stronger repulsive interactions between nanospheres, and as a result, the nanospheres could be more stable in an aqueous dispersion environment. In general, lipid nanospheres have a negative surface charge. Using $0.5 \%$ T- 80 and $0.5 \%$ lecithin as the surfactant, the nanosphere particles in sample $13 \mathrm{had}$ an increased zeta potential $(-26 \mathrm{mV})$ when 
compared with sample 8 (zeta potential $-15 \mathrm{mV}$ ) which contained $1 \% \mathrm{~T}-80$ as the surfactant.

For an optimized formulation intended for application to the skin, a small particle size, a low polydispersity index, and a higher zeta potential should be advantageous for skin penetration and drug loading. The sample 13 formulation was selected as a carrier for loading paeonol, in which $60 \%$ Dynasan 116 and 40\% Miglyol 812 was used as the solid and liquid lipid materials, respectively, and 0.5\% T-80 and $0.5 \%$ lecithin was used as the surfactant.

\section{Characterization of paeonol-loaded nanospheres}

TEM studies were carried out to gain further insight into the morphology of the paeonol-loaded nanospheres. TEM imaging of this formulation (Figure 3A and B) showed that the nanospheres had an almost spherical and homogeneous shape, with some irregularities at the periphery of the particles. The size of the paeonol-loaded nanospheres was also determined using a laser particle size analyzer, which demonstrated that each paeonol-loaded nanosphere was around $211 \mathrm{~nm}$ in diameter with a polydispersity index of $0.252 \pm 1.6$ (Figure 3D). The presence of paeonol molecules did not significantly change the particle size or zeta potential. The paeonol entrapment efficiency in the nanospheres was $76.9 \% \pm 2.1 \%$. Release of paeonol from the nanospheres was observed under sink conditions, and dynamic dialysis was used to separate the free drug from the paeonol-loaded nanospheres. Figure 3E shows a sustained release profile for the paeonol nanosphere. In contrast with release of paeonol from solution, whereby more than $90 \%$ of the paeonol is released within 8 hours (Figure 3C), release of paeonol from the nanosphere formulation was around $15 \%$ in the initial 3 hours, with approximately $70 \%$ of the paeonol having been released in a sustained manner by 72 hours. Because the paeonol entrapped in a solid and liquid lipid matrix needs time to diffuse from the lipid matrix to the surface and undergo partitioning between the lipid and aqueous phase, the nanospheres enabled prolonged release of paeonol.

\section{Optimization of hydrogel carrier for salidroside}

Three different types of gel-forming polymers, ie, Carbopol 940 , Carbopol 941, and xanthan gum, were prepared using an optimal stabilizing combination of water, and gel-forming polymer as a hydrating agent for encapsulation of salidroside. Carbopol 940 could not form a proper gel system incorporating nanospheres. Using Carbopol 941 and xanthan gum as carriers, the nanosphere-loaded gel samples were stable and showed
A

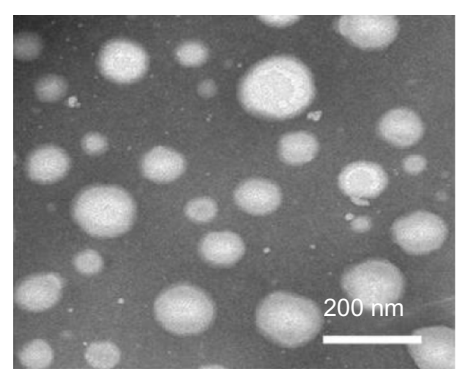

B

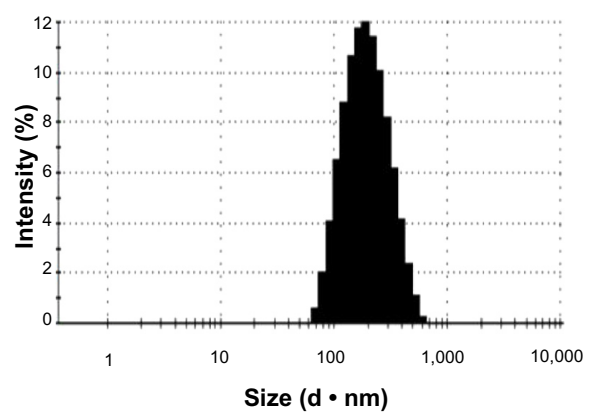

C

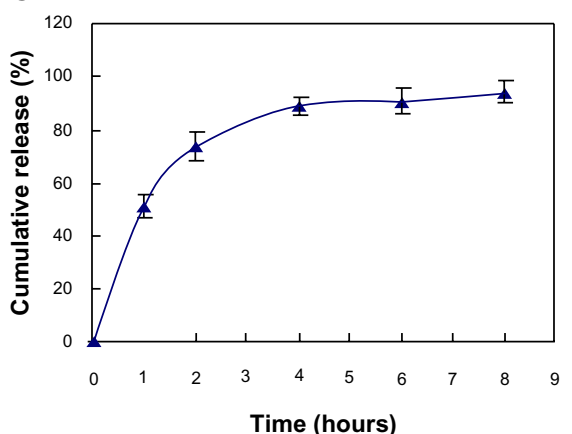

D Characterization of the nanospheres

\begin{tabular}{ll}
\hline Paeonol loaded NLCs & Parameters \\
\hline Size & $211 \pm 10 \mathrm{~nm}$ \\
PDI & $0.253 \pm 0.06$ \\
Zeta potential & $-29 \pm 3$ \\
Encapsulation & $76.92 \% \pm 5.15 \%$ \\
\hline
\end{tabular}

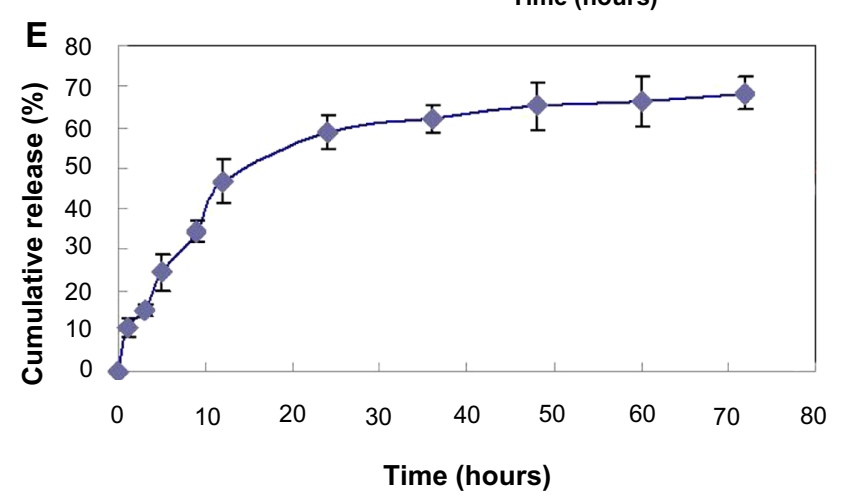

Figure 3 (A) Paeonol-loaded nanospheres observed by transmission electron microscopy. (B) Size distribution of the paeonol nanospheres. (C) Cumulative release of paeonol from solution. (D) Characterization of the paeonol nanosphere dispersion. (E) Cumulative release profile for paeonol in the nanosphere dispersion. Abbreviations: NLCs, nanostructured lipid carriers; PDI, polydispersity index. 


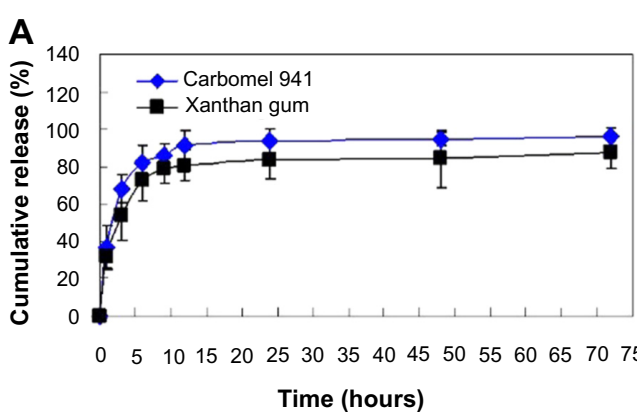

D

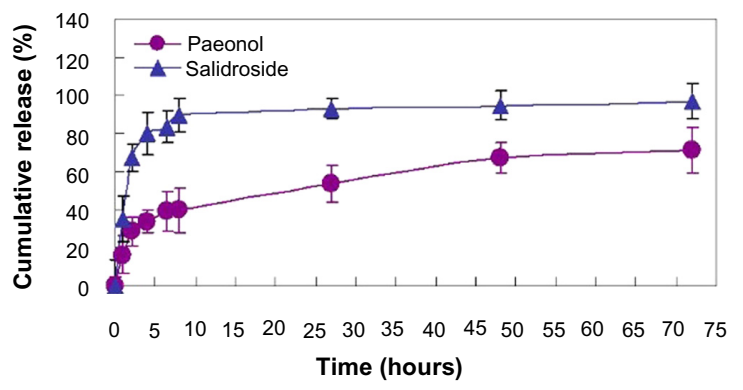

B

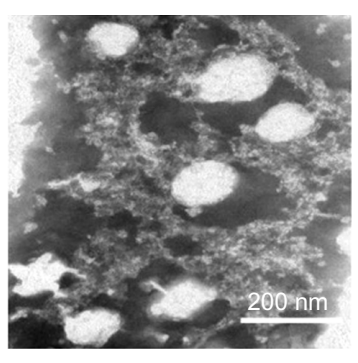

$\mathbf{E}$
C Characterization of the nanosphere-gel

Paeonol and salidroside co-loaded nanosphere-gel

Nanosphere size $\quad 286 \pm 14 \mathrm{~nm}$

Nanosphere PDI $\quad 0.397 \pm 0.11$

Nanosphere zeta potential $\quad-22$

Paeonol encapsulation $\quad 65.33 \%+2.26 \%$

Hydrogel $\mathrm{pH} \quad 6.7 \pm 0.3$

Hydrogel viscosity $\quad 16 \pm 0.2 \mathrm{pa} \cdot \mathrm{S}$

Figure 4 (A) Cumulative release profiles for salidroside in the hydrogel formulation. (B) Paeonol nanospheres in the hydrogel system observed by transmission electron microscopy. (C) Characterization of the paeonol and salidroside incorporated into the nanosphere gel system. (D) Cumulative release profiles for salidroside and paeonol in the nanosphere-gel system. (E) Size and polydispersity index of the nanospheres in the gel system over 4 weeks.

Abbreviation: PDI, polydispersity index.

little sedimentation even after centrifugation. Figure 4A shows that the sustained release profiles for salidroside from the Carbopol hydrogel and from the xanthan gum are similar. However, because xanthan gum has a more sticky texture, the cumulative percent release of salidroside from the Carbopol 941 gel was greater than that from the xanthan gum. In the first 24 hours, around $91 \%$ of the salidroside was released from the Carbopol 941 hydrogel, with almost all the loaded drug $(95.3 \%)$ released in about 72 hours. In contrast, 83.6\% and $87.9 \%$ of the salidroside was released from the xanthan gum at 24 hours and 72 hours. Carbopol 941 hydrogel was also better in appearance and texture, so was selected as the carrier for rapid release and delivery of salidroside. Further, Carbopol 941 hydrogel is thermally stable and has optimum rheological properties for skin application.

\section{Characterization of the sequential release paeonol-salidroside nanosphere-gel system}

Carbopol 941 gel loaded with the nanospheres had a smooth appearance. As shown in Figure 4B and C, the size of the paeonol nanospheres in the Carbopol 941 gel was $286 \mathrm{~nm}$ (polydispersity index 0.397), indicating that they could maintain a spherical and homogeneous shape in the gel system. The final $\mathrm{pH}$ of the nanosphere-gel was adjusted to around 6.7 and its viscosity was around $16 \mathrm{~Pa} \cdot \mathrm{S}$. These results suggest that the prepared hydrogel can supply an advantageous topical carrier for the nanosphere application.

The cumulative percent release of paeonol from the nanosphere-gel dispersion was investigated over a period of 72 hours. Figure 4D shows that approximately $30 \%$ of the paeonol was released in the initial 2 hours due to slow diffusion of drug from the lipid. After 2 hours, the drug release rate increased. The liquid lipid-enriched nanospheres were soft and enabled considerably higher solubility of lipophilic paeonol, in which the drug was easily loaded to a higher amount and paeonol could be easily released as well by the drug diffusion or the matrix erosion manners. Compared with the drug-release profile for the nanosphere suspension (Figure 3E), release of paeonol from the nanosphere-gel was sustained by the gel system. However, by 72 hours, drug release from the nanosphere suspension and nanosphere-gel were $54.57 \%$ and $64.31 \%$, respectively, further indicating that the external phase of the hydrogel is advantageous for sustained release of paeonol by decreasing the cumulative percent release. At the same time, the release of salidroside from the nanospheres was only slightly affected by incorporation of paeonol-nanospheres into the hydrogel.

\section{Stability of nanosphere-hydrogel}

The particle size of the nanospheres in the Carbopol-gel system was monitored for up to 4 weeks at $4^{\circ} \mathrm{C}$. After 4 
weeks of storage, the particle size remained in the range of $276-457 \mathrm{~nm}$ and the polydispersity index remained below 0.36 (Figure 4E), indicating that the nanospheres were stable in hydrogel over this time.

\section{Melanogenesis in UVB-irritated guinea pig skin}

As detailed in the Materials and methods section, the guinea pigs were exposed to UVB light once daily for 2 consecutive weeks. UVB light exposure was then stopped and the skin was observed without any handling for a further 2 weeks. Figure 5 shows no apparent change in the skin, with no pigmentation observed on day 7 post-irradiation (Figure 5A). On day 14, some melanin is apparent in the central area, and some obscure pigment spots were observed in the surrounding area (Figure 5B). On day 21, thicker pigment can be observed in both the central and peripheral areas (Figure 5C). On day 28, darker pigment is clearly observed and black spots are distributed throughout the irradiated skin area. These results suggest that the pigmentation process was more active in the 2 weeks after UVB irradiation than during the period of irradiation itself, and that there was a delay between UVB irradiation and peak melanin synthesis. In a previous study, we applied topical paeonol and salidroside solutions to UVB-irradiated skin, and found that these preparations could only decrease melanin synthesis initiated by UVB irradiation. Because nanoparticle-based preparations form a "film" covering the skin and may be used as an UV absorber, in this study, all the drug-loaded preparations or hybrid systems were applied simultaneously with UVB irradiation.

\section{Effect of sequential release of paeonol and salidroside from nanosphere-gel on UVB-induced melanogenesis}

Figure 6 shows the skin gloss appearance (line I), and the microscopic appearance of the epidermal (line II) and dermal (line III) layers. In contrast with the blank control, there was a visible decrease in skin tanning in all groups treated with the drug-loaded preparations. As shown in Figure 6A,

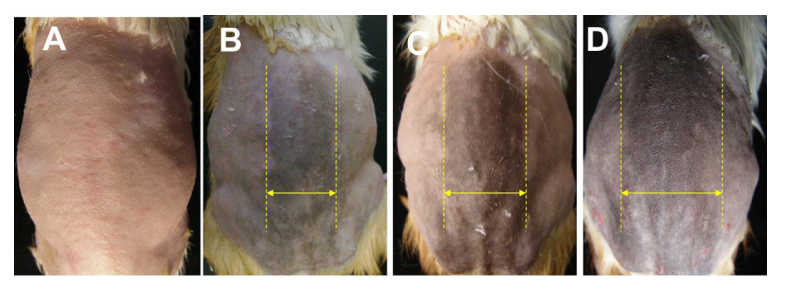

Figure 5 Photographs of skin appearance. (A) Day 7 post-irradiation. (B) Day 14 post-irradiation. (C) Day 21 post-irradiation. (D) Day 24 post-radiation. The hyperpigmentation area is indicated with yellow dashed lines and arrows. the pigment was disposed and obvious pigment spots were observed in most areas of the skin after UVB irradiation without any treatment, while pigmentation seemed to be decreased in the other treated groups (Figure 6B-D). Further, the skin had a lighter color after treatment with the paeonol and salidroside dual-release nanosphere-hydrogel system (Figure 6D) than in the groups treated with the salidrosidegel (Figure 6B) or paeonol-nanosphere (Figure 6C) systems. The hyperpigmented skin specimens were also analyzed by hematoxylin and eosin staining, the results of which indicated significantly condensed pigment in the epidermis of the blank control (vehicle) group when compared with the three active treatment groups (Figure D). The skin pigments were identified using the melanin-specific Fontana-Masson stain. Figure $6 \mathrm{E}$ confirms that the melanin in all the three drug-loaded preparation treated groups was significantly decreased when compared with the control group. Further, the melanin content in skin treated with the paeonol and salidroside sequential-release nanosphere-gel was clearly lower than that in skin treated with preparations containing only paeonol or salidroside.

\section{Discussion}

Although melanin protects the skin from damage induced by UV light, abnormal hyperpigmentation caused by increased production of melanin can cause skin disorders as well as psychosocial and cosmetic concerns. ${ }^{38,39}$ The availability of depigmenting agents is presently rather limited because of their high toxicity, low stability, poor skin penetration, and insufficient activity. ${ }^{40}$ For example, kojic acid, a tyrosinase inhibitor, has been the subject of great interest in the cosmetic industry because of its anti-pigmenting effects. ${ }^{41}$ However, synthetic agents often result in skin inflammation, have insufficient penetration, or have undefined clinical efficiency, so alternatives are being sought. ${ }^{42}$ As a result, much effort has been devoted to developing preparations with plant or herbal components for the treatment of pigmentation disorders and as skin-whitening cosmetic agents. ${ }^{43-45}$ In a previous study, we demonstrated the potential of two natural products, ie, salidroside and paeonol, to achieve depigmentation by inhibiting the proliferation of melanocytes in the basal epidermal layer and decreasing tyrosinase activity. However, application of these compounds is greatly hampered by their low stability, poor skin penetration, and potential for toxicity at high dosages. ${ }^{46-49}$ Additionally, because stimulation of melanocyte proliferation and tyrosinase activity are two major mechanisms for the increased melanin synthesis caused by UVB radiation, it is 

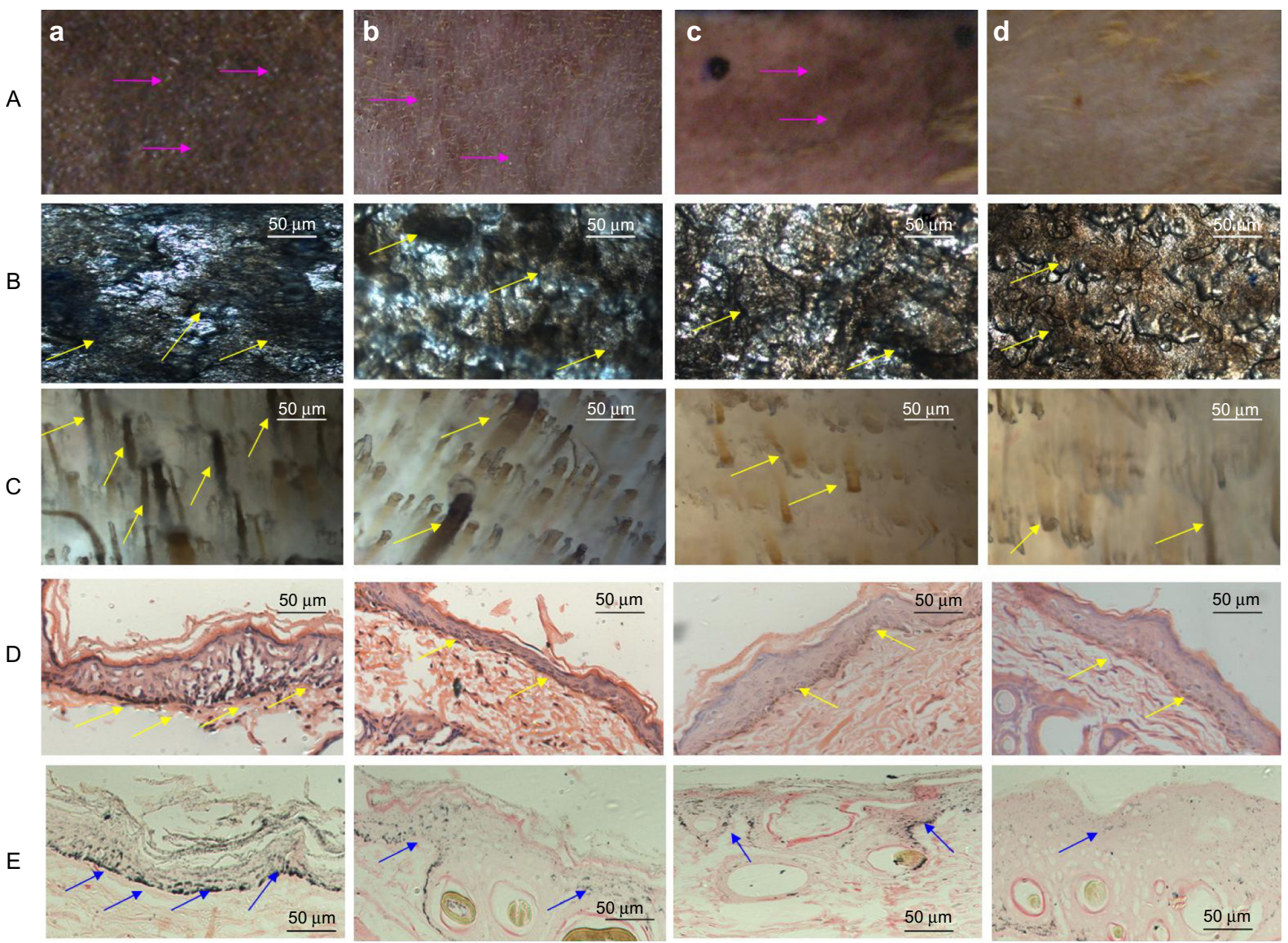

Figure 6 Effects of the drug preparations on hyperpigmentation in guinea pig skin stimulated by 2 weeks of UVB radiation. (A) Photographs of the guinea pig skin appearance (obvious pigment indicated by purple arrows). (B) Microscopic appearance of the pigmented epidermis (obvious pigment indicated by yellow arrows). (C) Microscopic appearance of the pigmented dermis (obvious pigment indicated by yellow arrows). (D) Hematoxylin and eosin-stained images of pigmented skin sections (obvious pigment indicated by yellow arrows). (E) Fontana-Masson stain images of melanin in the epidermal layer (obvious melanin indicated by blue arrows). (a) Blank control, (b) group treated with salidroside-loaded hydrogel, (c) group treated with paeonol-loaded nanosphere dispersion, and (d) group treated with the sequential-release salidroside and paeonol nanosphere-gel.

Abbreviation: UVB, ultraviolet $B$.

hypothesized that a nanosphere-gel system enabling burst release of salidroside and sustained release of paeonol may rapidly inhibit UVB-induced melanogenesis and remove any melanin formed in a sustained manner.

Therefore, in this study, a nanosphere-gel formulation enabling sequential release of salidroside and paeonol was prepared and investigated for its effects on depigmentation in vivo. First, the lipid nanospheres were optimized according to type and ratio of lipid materials, and it was found that Dynasan 116 and Miglyol 812 at a ratio of 6:4 was the best for the nanosphere formulation. It was also found that a combination of $0.5 \% \mathrm{~T}-80$ and $0.5 \%$ lecithin as the surfactant for the nanosphere formulation had the advantages of decreasing the particle size and polydispersity index of the nanospheres and increasing their stability. No agglomeration or change in particle size was detected for the nanospheres in hydrogel during 4 weeks of storage at $4^{\circ} \mathrm{C}$. With this formulation, the loaded paeonol can be entrapped by $65.7 \%$, indicating that most of the paeonol could be entrapped into the nanospheres. The reason for this high entrapment efficiency may be that the mixture of liquid and solid lipids has more positions available for occupation by lipid-soluble paeonol. ${ }^{50}$ Therefore, the lipid nanospheres were dispersed in a hydrogel system incorporating salidroside without inducing dissolution or aggregation of the nanospheres. ${ }^{51}$ It was shown that introduction of nanospheres containing therapeutic molecules into hydrogels allowed rapid delivery of salidroside from the hydrogel followed by sustained release of paeonol. Approximately $36.9 \%$ of the salidroside was released efficiently from the delivery system in the first hour, with over $91 \%$ released over 12 hours, indicating a burst effect which could help to prevent proliferation of melanocytes and subsequent melanogenesis. Also, $67 \%$ of the paeonol loaded in the nanospheres was released in a sustained manner over 72 hours, allowing prolonged inhibition of the pathological 
tyrosinase activity occurring in melanocytes that had not been suppressed by salidroside.

The efficacy of this dual drug-releasing nanosphere-gel system in preventing UVB-induced melanogenesis was investigated in the brown guinea pig. The nanosphere-hydrogel system loaded with salidroside and paeonol was more effective for decreasing melanin levels in the UVB-stimulated guinea pig skin than the treatments received by the other groups. Histological analysis and specific Fontana-Masson staining showed that hyperpigmentation treated with the hybrid system was significantly improved compared with that in the groups receiving the blank control or single-drug loaded preparations.

From the hematoxylin and eosin and Fontana-Masson staining experiments, it is clear that the most important mechanisms for protection of skin from UV rays, ie, thickening of the stratum corneum and synthesis of melanin by melanocytes, were decreased by the dual drug-releasing system. The sequential delivery of multiple depigmentation molecules with different therapeutic functions was shown to be a promising strategy enhancing the therapeutic efficacy of the study drugs. Mechanistically, as indicated by the results from the animal model, it was observed that after 2 weeks of UVB radiation, the black spots with highest density were observed at weeks 3-4 after cessation of UVB radiation. These phenomena indicate that there is a delay between UVB radiation and peak melanin synthesis. The pathological melanin synthesis activated by proliferating melanocytes and tyrosinase activity is a sustained process lasting for a long period of time. Therefore, the enhanced anti-melanogenesis effect expressed by the nanosphere-gel may be contributed to that the burst release of salidroside incorporated in the hydrogel can give a prompt inhibition for the melanocytes proliferation, while sustained release of paeonol provides continuous inhibition of tyrosinase activity in melanocytes.

Further, in contrast with the strategy of delivering multiple drugs simultaneously to enhance their therapeutic effect, ${ }^{52}$ the use of a hybrid system releasing drugs sequentially may avoid delivery of excessive doses and side effects can be reduced. In the present study, as the dosage for each time of all the drug-treated groups is the same as $0.2 \mathrm{mg}$, which is not a sufficiently high dosage to induce obvious side effects in a short period, the advantage of the sequential release of salidroside and paeonol in decreasing the side effects and toxicity of drugs may be not indicated obviously by the results presented in this study However, the strategy of sequential drug release is thought to be a very useful method for decreasing the doses of individual drugs and avoiding the toxicity seen when they are used at high doses and/or for a long period of time.

In summary, the results of this study clearly indicate that timely delivery of multiple drugs in sequence is a promising strategy for the treatment of melanogenesis. In addition to use of multiple natural products to decrease melanogenesis as demonstrated in this study, various combinations of proteins and genes could be also loaded into this hybrid nanospheregel delivery system for many therapeutic purposes. Our approach of designing and tailoring delivery systems to release multiple therapeutic molecules in a sequential and sustained manner is expected to have wide application in nanomedicine.

\section{Conclusion}

The sequential release of salidroside and paeonol by a nanosphere-gel system was shown to significantly enhance the anti-melanogenesis effects of these agents and may reduce their potential for toxicity when used in high doses. The nanosphere-hydrogel system was demonstrated to be a promising carrier for the delivery of multiple drugs in an orchestrated manner, with great potential in nanomedicine.

\section{Acknowledgments}

This research was supported by the National Natural Science Foundation of China (grant 81102393), the 48th China Postdoctoral Science Foundation (grant 420000-X91004), the Zhejiang Provincial Program for the Cultivation of High-Level Innovative Health Talents, and the Fundamental Research Funds for the Central Universities, People's Republic of China.

\section{Disclosure}

The authors report no financial or other conflicts of interest relevant to the subject of this paper.

\section{References}

1. Costin GE, Hearing VJ. Human skin pigmentation: melanocytes modulate skin color in response to stress. FASEB J. 2007;21:976-994.

2. Yamaguchia Y, Nagasawa T, Nakamura N, et al. Successful treatment of photo-damaged skin of nano-scale atRA particles using a novel transdermal delivery. J Control Release. 2005;104:29-40.

3. Akeo K, Amaki S, Suzuki T, Hiramitsu T. Melanin granules prevent the cytotoxic effects of L-DOPA on retinal pigment epithelial cells in vitro by regulation of $\mathrm{NO}$ and superoxide radicals. Pigment Cell Res. 2000;13:80-88.

4. Schallreuter KU, Hordinsky MK, Wood JM. Thioredoxin reductase. Role in free radical reduction in different hypopigmentation disorders. Arch Dermatol. 1987;123:615-619.

5. Altaei T. The treatment of melasma by silymarin cream. BMC Dermatol. 2012;12:18. 
6. Del BS, Bernerd F. Relationship between skin color and skin response to ultraviolet light. Int J Dermatol. 2012;Suppl 1:12-18.

7. Chen N, Hu Y, Li WH, Eisinger M, Seiberg M, Lin CB. The role of keratinocyte growth factor in melanogenesis: a possible mechanism for the initiation of solar lentigines. Exp Dermatol. 2010;19:865-872.

8. Chapman MS, Quitadamo MJ, Perry AE. Pigmented squamous cell carcinoma. J Cutan Pathol. 2000;27:93-95.

9. Li N, Peng LH, Chen X, et al. Transcutaneous vaccines: novel advances in technology and delivery for overcoming the barriers. Vaccine. 2011;29:6179-6190.

10. Stratigos AJ, Forsea AM, van der Leest RJ, et al. Euromelanoma: a dermatology-led European campaign against nonmelanoma skin cancer and cutaneous melanoma. Past, present and future. Br J Dermatol. 2012;167 Suppl 2:99-104.

11. Yanase H, Ando H, Horikawa M, Watanabe M, Mori T, Matsuda N. Possible involvement of ERK 1/2 in UVA-induced melanogenesis in cultured normal human epidermal melanocytes. Pigment Cell Res. 2001:14:103-109.

12. Abdel-Malek ZA, Kadekaro AL, Swope VB. Stepping up melanocytes to the challenge of UV exposure. Pigment Cell Res. 2010;23:171-186.

13. Solano F, Briganti S, Picardo M, et al. Hypopigmenting agents: an updated review on biological, chemical and clinical aspects. Pigment Cell Res. 2006;19:550-571.

14. Rees JL. Genetics of hair and skin color. Annu Rev Genet. 2003;37: 67-90.

15. Regad T. Molecular and cellular pathogenesis of melanoma initiation and progression. Cell Mol Life Sci. 2013;70:4055-4065.

16. Ravnbak MH, Philipsen PA, Wiegell SR, Wulf HC. Skin pigmentation kinetics after UVB exposure. Acta Derm Venereol. 2008;88:223-228.

17. Dynoodt P, Mestdagh P, Van Peer G, et al. Identification of miR-145 as a key regulator of the pigmentary process. J Invest Dermatol. 2013;133:201-209.

18. Peng H, Liu S, Xu SY, et al. Inhibitory effects of salidroside and paeonol on tyrosinase activity and melanin synthesis in mouse B16F10 melanoma cells and ultraviolet B-induced pigmentation in guinea pig skin. Phytomedicine. 2013;20:1082-1087.

19. Shi J, Ma F, Wang X, et al. Formulation of liposome gels of paeonol for transdermal drug delivery by Box-Behnken statistical design. J Liposome Res. 2012;22:270-278.

20. Luo, Shen Q, Chen J. Transdermal delivery of paeonol using cubic gel and microemulsion gel. Int J Nanomedicine. 2011;6:1603-1610.

21. Peng H, Dong R, Wang S, et al. A pH-responsive nano-carrier with mesoporous silica nanoparticles cores and poly(acrylic acid) shelllayers: fabrication, characterization and properties for controlled release of salidroside. Int J Pharm. 2013;446:153-159.

22. Verma P, Pathak K. Nanosized ethanolic vesicles loaded with econazole nitrate for the treatment of deep fungal infections through topical gel formulation. Nanomedicine. 2012;8:489-496.

23. de Paula E, Cereda CM, Fraceto LF, et al. Micro and nanosystems for delivering local anesthetics. Expert Opin Drug Deliv. 2012;9: $1505-1524$.

24. Basnet P, Škalko-Basnet N. Nanodelivery systems for improved topical antimicrobial therapy. Curr Pharm Des. 2013;19:7237-7243.

25. Peng H, Wei W, Qi XT, et al. Epidermal stem cells manipulated by pDNA-VEGF165/CYD-PEI nanoparticles loaded gelatin/ $\beta$-TCP matrix as therapeutic agent and gene delivery vehicle for wound healing. Mol Pharm. 2013;10:3090-3102.

26. Friedman AJ, Phan J, Schairer DO, et al. Antimicrobial and antiinflammatory activity of chitosan-alginate nanoparticles: a targeted therapy for cutaneous pathogens. J Invest Dermatol. 2013;133: 1231-1239.

27. Chen X, Peng LH, Shan YH, et al. Astragaloside IV-loaded nanoparticleenriched hydrogel induces wound healing and anti-scar activity through topical delivery. Int J Pharm. 2013;447:171-181.

28. Hadinoto K, Sundaresan A, Cheow WS. Lipid-polymer hybrid nanoparticles as a new generation therapeutic delivery platform: a review. Eur J Pharm Biopharm. 2013;85(3 Pt A):427-443.
29. Yang H, Tyagi P, Kadam RS, et al. Hybrid dendrimer hydrogel/PLGA nanoparticle platform sustains drug delivery for one week and antiglaucoma effects for four days following one-time topical administration. ACS Nano. 2012;6:7595-7606.

30. Pradhan, Singh D, Singh MR. Novel colloidal carriers for psoriasis: current issues, mechanistic insight and novel delivery approaches. J Control Release. 2013;170:380-395.

31. Fan X, Chen J, Shen Q. Docetaxel-nicotinamide complex-loaded nanostructured lipid carriers for transdermal delivery. Int $J$ Pharm. 2013;458:296-304.

32. Nishizaka T, Nomura T, Sano T, Higuchi K, Nagatomo F, Ishihara A. Hyperbaric oxygen improves ultraviolet B irradiation-induced melanin pigmentation and diminishes senile spot size. Skin Res Technol. 2011;17(3):332-338.

33. Stout GJ, Blasco MA. Telomere length and telomerase activity impact the UV sensitivity syndrome xeroderma pigmentosum C. Cancer Res. 2013;73:1844-1854.

34. Hoshino T, Matsuda M, Yamashita Y, et al. Suppression of melanin production by expression of HSP70. J Biol Chem. 2010;285: 13254-13263.

35. Kim JH, Min KH, Heo CY, et al. Histological evaluation of dermal tissue remodeling with the 1444-nm neodymium:yttrium-aluminumgarnet laser in in vivo model. $J$ Dermatol. 2013;40:706-710.

36. Kabashima K, Sakabe JI, Yoshiki R, Tabata Y, Kohno K, Tokura Y. Involvement of Wnt signaling in dermal fibroblasts. Am J Pathol. 2010;176:721-732.

37. Bao YP, An W, Turner CH, et al. The critical role of surfactants in the growth of cobalt nanoparticles. Langmuir. 2010;26:478-483.

38. Curto EV, Kwong C, Hermersdörfer $\mathrm{H}$, et al. Inhibitors of mammalian melanocyte tyrosinase: in vitro comparisons of alkyl esters of gentisic acid with other putative inhibitors. Biochem Pharmacol. 1999;57:663-672.

39. Burnett CL, Bergfeld WF, Belsito DV, et al. Final report of the safety assessment of kojic acid as used in cosmetics. Int J Toxicol. 2010; 29(Suppl 6):244S-273S

40. Chang TS. An updated review of tyrosinase inhibitors. Int J Mol Sci. 2009; 10:2440-2475.

41. Pieroni A, Quave CL, Villanelli ML, et al. Ethnopharmacognostic survey on the natural ingredients in folk cosmetics, cosmeceuticals and remedies skin disease in the inland Marches, Central-Eastern Italy. J Ethnopharmacol. 2004;91:331-344.

42. Momtaz S, Mapunya BM, Houghton PJ, et al. Tyrosinase inhibition by extracts and constituents of Sideroxylon inerme L. stem bark, used in South Africa for skin lightening. J Ethnopharmacol. 2008;119:507-512.

43. Jang JY, Lee JH, Jeong SY, Chung KT, Choi YH, Choi BT. Partially purified Curcuma longa inhibits alpha-melanocyte-stimulating hormone-stimulated melanogenesis through extracellular signalregulated kinase or Akt activation-mediated signalling in $\mathrm{B} 16 \mathrm{~F} 10$ cells. Exp Dermatol. 2009;18:689-694.

44. Bao MH, Zhang YW, Zhou HH. Paeonol suppresses oxidized lowdensity lipoprotein induced endothelial cell apoptosis via activation of LOX-1/p38MAPK/NF-אB pathway. J Ethnopharmacol. 2013;146:543-551.

45. Li H, Wang S, Zhang B, et al. Influence of co-administered danshensu on pharmacokinetic fate and tissue distribution of paeonol in rats. Planta Med. 2012;78:135-140.

46. Zhu J, Wan X, Zhu Y, Ma X, Zheng Y, Zhang T. Evaluation of salidroside in vitro and in vivo genotoxicity. Drug Chem Toxicol. 2010;33: 220-226.

47. Zhang H, Shen WS, Gao CH, Deng LC, Shen D. Protective effects of salidroside on epirubicin-induced early left ventricular regional systolic dysfunction in patients with breast cancer. Drugs R D. 2012;12: 101-106.

48. Wang XL, Wang X, Xiong LL, et al. Salidroside improves doxorubicininduced cardiac dysfunction by suppression of excessive oxidative stress and cardiomyocyte apoptosis. J Cardiovasc Pharmacol. 2013;62: $512-523$. 
49. Li D, Fu Y, Zhang W, et al. Salidroside attenuates inflammatory responses by suppressing nuclear factor- $\kappa \mathrm{B}$ and mitogen activated protein kinases activation in lipopolysaccharide-induced mastitis in mice. Inflamm Res. 2013;62:9-15.

50. Zhang WL, Gu X, Bai H, Yang RH, Dong CD, Liu JP. Nanostructured lipid carriers constituted from high-density lipoprotein components for delivery of a lipophilic cardiovascular drug. Int J Pharm. 2010;391:313-321.
51. Bhaskar K, Mohan CK, Lingam M, Mohan SJ, Venkateswarlu V, Rao YM. Development of SLN and NLC enriched hydrogels for transdermal delivery of nitrendipine: in vitro and in vivo characteristics. Drug Dev Ind Pharm. 2009;35:98-113.

52. Yu JS, Kim AK. Effect of combination of taurine and azelaic acid on antimelanogenesis in murine melanoma cells. J Biomed Sci. 2010; 17 Suppl 1:S45-S49.

\section{Publish your work in this journal}

The International Journal of Nanomedicine is an international, peerreviewed journal focusing on the application of nanotechnology in diagnostics, therapeutics, and drug delivery systems throughout the biomedical field. This journal is indexed on PubMed Central, MedLine, CAS, SciSearch ${ }^{\circledR}$, Current Contents ${ }^{\circledR} /$ Clinical Medicine,
Journal Citation Reports/Science Edition, EMBase, Scopus and the Elsevier Bibliographic databases. The manuscript management system is completely online and includes a very quick and fair peer-review system, which is all easy to use. Visit http://www.dovepress.com/ testimonials.php to read real quotes from published authors. 